\section{Abstract}

\title{
Owner and veterinary surgeon perspectives on the roles of veterinary nurses and receptionists in relation to small animal preventative healthcare consultations in the United Kingdom.
}

\author{
Zoe Belshaw, Natalie J Robinson, Rachel S Dean and Marnie L. Brennan
}

Centre for Evidence-based Veterinary Medicine, School of Veterinary Medicine and Science, University of Nottingham Sutton Bonington Campus LE12 5RD

Corresponding author: M.L. Brennan: marnie.brennan@nottingham.ac.uk

\begin{abstract}
Veterinary receptionists and veterinary nurses rarely feature in published practice-based research, yet are integral to small animal veterinary practice in the United Kingdom (UK). The aim of this study was to investigate the perspectives of UK-based owners and veterinary surgeons about veterinary nurses and receptionists in relation to their role in preventative healthcare. Semi-structured telephone interviews were conducted with $15 \mathrm{dog}$ and cat owners and 14 veterinary surgeons. Interview transcripts were thematically analysed. Reception staff were identified as having a range of important roles, from rapport building to providing healthcare information and advice. The perceived importance of those roles appeared to differ between owners and veterinary surgeons. Veterinary nurses were described as performing a diversity of roles in relation to preventative healthcare, both in the reception area and in the consulting room. Many owners, and some veterinary surgeons, expressed uncertainty about the remit and status of veterinary nurses in relation to providing veterinary advice. This study identifies for the first time the degree of responsibility for preventative healthcare given to veterinary receptionists and veterinary nurses in UK small animal practices. Further work is needed involving reception and nursing staff to fully appreciate and define their roles in small animal practice.
\end{abstract}

Keywords: preventative healthcare; dog; cat; veterinary receptionist; veterinary nurse.

\section{Introduction}

Veterinary nurses and receptionists working in small animal practice are seldom the primary subject of peer-reviewed research. Historically, they were termed "support staff". ${ }^{1}$ However, Kinnison and others ${ }^{2}$ advocate the use of the term "inter-professional practice" when describing their role within a veterinary team, reflecting their importance as true partners in delivering care. The largest body of published research to include veterinary nurses and receptionists has focused on the importance of, and tensions related to, integrating them into a veterinary team. ${ }^{2-5}$

Alongside traditional roles in health, wellbeing and as guardians of animal welfare ${ }^{6}$, veterinary nurses now have a range of responsibilities. These include performing consultations ${ }^{7}$ and minor surgeries ${ }^{2}$, running educational interventions ${ }^{8}$ and providing complex inpatient care. ${ }^{9}$ Other less commonly documented roles include building rapport, facilitating good communication and providing empathy in a client-facing setting. ${ }^{10,11}$ In many small animal practices, veterinary nurses may also perform the duties of receptionist, practice manager and cleaner. ${ }^{12}$

The veterinary nursing profession in the United Kingdom (UK) has moved towards increasing status and regulation in recent years. ${ }^{2}$ Veterinary nurses registered with the Royal College of Veterinary Surgeons are now accountable for their own conduct ${ }^{13}$ and are subject to standards set by the 
Veterinary Nursing Council. ${ }^{14}$ However, despite a vigorous campaign, the title of "veterinary nurse" is still not protected. ${ }^{15}$ Some veterinary practices may therefore employ other staff under a range of titles who share some of the client-facing nursing responsibilities described above without the same training or regulatory accountability.

45 If little has been written about the role of the veterinary nurse, even less is written about veterinary receptionists. Veterinary receptionists are not required to have any formal training and are not regulated. Despite this, they have an incredibly important and responsible role as gatekeepers of the veterinary practice and as the hub of practice communication. ${ }^{3}$ They are also central to the customer experience, providing information and steering the client through their interactions with the practice. ${ }^{16}$ Increasingly, their roles and responsibilities are becoming broader, an example being provision of advice on preventative medicine protocols. ${ }^{17}$ For these reasons, the role of a veterinary receptionist appears challenging, and there is a risk that they could become scapegoats for mistakes in communication if expectations about their knowledge do not reflect their training or experience. ${ }^{3}$

Soliciting the views of the people who interact with these staff may help to identify previously unrecognised roles and responsibilities and may highlight unreported challenges to their work. Qualitative research techniques such as interviews and focus groups are particularly useful for this type of exploratory research. The aim was to explore the perspectives of owners and veterinary surgeons regarding veterinary nurses and receptionists, by eliciting accounts of their interactions with these staff members in relation to small animal (dog and cat) preventive medicine consultations.

\section{Materials and methods}

This study forms part of a larger body of work exploring canine and feline preventative medicine consultations in the UK. During July and August 2016, data were gathered through telephone interviews with dog and/or cat owners and small animal veterinary surgeons based in the UK. Ethical approval for this study was granted by the ethics committee at the School of Veterinary Medicine and Science, University of Nottingham. Reporting follows the COREQ checklist. ${ }^{18}$

\section{Owner and veterinary surgeon recruitment}

Owner and veterinary surgeon recruitment for interview was based on separate purposive sampling frames designed by the authors (see Tables 1 and 2 in supplementary materials). These sampling frames were used as described by Bryman ${ }^{19}$ to try to include a wide variety of interviewees in the study. The owner sampling frame included pet, owner and practice variables; the veterinary surgeon version included practice and veterinary surgeon variables.

Inclusion criteria for owner interviewees were: a) ownership of at least one dog or cat that had received a preventative healthcare consultation at a UK veterinary practice during the preceding 3 months; AND b) willingness to be interviewed by telephone about that consultation during the study period. Eligible consultations were: routine vaccination; titre testing; parasite prevention; routine health check; or prevention of season. These consultation types were chosen based on previous data ${ }^{20}$ reporting the characteristics of consultations where preventative medicine was most likely to be discussed, and where a complete clinical examination was likely to be performed. Inclusion criteria for veterinary surgeons were: a) individuals currently working in a UK small animal practice; AND b) who currently performed preventative healthcare consultations; AND c) were available for telephone interview during the study period. 
Owners were recruited using: direct contact of eligible participants from the authors' networks; social media posts in owner forums; recruitment of eligible clients by veterinary surgeons in a multibranch veterinary practice in Scotland; and snowball sampling whereby recruited interviewees assisted with recruitment. ${ }^{19}$ Veterinary surgeon recruitment was conducted using: social media; contacting veterinary practices who had expressed an interest in research collaboration with the Centre for Evidence-based Veterinary Medicine; emails to the Royal College of Veterinary Surgeons' list of practices; and snowball sampling.

\section{Interview procedure}

Interested owners and veterinary surgeons were emailed information about the study and a copy of the consent form. Those willing to proceed were asked to supply information to confirm their eligibility before a date was arranged for an interview. Incentives to participate were not offered. All telephone interviews were conducted by NR, a veterinary surgeon with qualitative research training. Before each interview, the consent form was read in full and verbal consent to proceed was granted. Separate semi-structured interview guides pilot-tested before use (available on request), were used for owner and veterinary surgeon interviews. Experiences with all preventative healthcare consultations during the preceding 3 months were discussed with the owners, and attitudes to preventative healthcare consultations in general were discussed with veterinary surgeons. Pertinent to this study, participants were asked about the roles of veterinary nurses and receptionists in preventative healthcare.

\section{Data analysis}

Interviews were recorded with a Dictaphone using a telephone adapter before being professionally transcribed verbatim. Transcripts were checked for accuracy against audio recordings and any discrepancies amended. Thematic analysis was performed following the six-step plan described by Braun and Clarke ${ }^{21}$ with the organisational support of nVivo (nVivo v11, QSR), described in greater detail elsewhere. ${ }^{22}$ Statistical analysis was not performed as is standard for qualitative studies. ${ }^{23,24}$ Themes were identified inductively and deductively. Data analysis was performed primarily by ZB, assisted through regular discussion with $\mathrm{MB}$. Both are veterinary surgeons with qualitative research experience. Data saturation was deemed to be achieved when no additional themes were identified as a result of analysing further transcripts.

\section{Results}

Thirty-one interviews were arranged, but two owners were unavailable due to unforeseen circumstances on the day of the interview. Twenty nine telephone interviews were conducted, 15 owners and 14 with veterinary surgeons. At this point, data saturation had been reached, so additional interviewees were not recruited. Full demographic details of owners, their pets, veterinary surgeons and their practices have previously been described. ${ }^{25}$ Interview duration ranged from 15 to 59 minutes. The nine female and five male veterinary surgeons were from 12 practices. All had graduated in the preceding 20 years and ranged in seniority from assistant to clinical director. Practice types included corporate and independent, small animal and mixed, single and multi-branch. Thirteen dog owners, one cat owner, and an owner of both a dog and a cat were interviewed. Between them, they owned 19 dogs and 3 cats which ranged in age from 6 months to 11 years. Dogs included pets, agility dogs and working gun dogs; cats lived both indoors and outdoors.

Thematic analysis deductively identified four key themes, each of which has been reported separately. These described: expectations of owners and veterinary surgeons about what would 
happen during preventative healthcare consultations ${ }^{25}$; the importance of the length of preventative healthcare consultations ${ }^{22}$; motivators and barriers to using preventative medicines ${ }^{26}$; and the role of veterinary nurses and receptionists in preventative healthcare. This last key theme is reported here with illustrative quotes. Interviewees were not provided with definitions of the terms "veterinary nurse" or "receptionist" so the use of those terms below reflect the descriptors used by interviewees.

\section{Owners}

Owners identified four settings in which they interacted with receptionists and/or veterinary nurses in relation to preventative healthcare: on the telephone; in the waiting room; during veterinary surgeon-led consultations; and during veterinary nurse-led consultations. Rarely, owners reflected that they were not sure of the job role, or level of training, of the person behind the reception desk.

Actually I felt unclear whether she was a nurse or reception staff. So I thought it was one of those roles where they probably learnt some things because I think I spoke to somebody when I was talking about the car sickness and she mentioned a couple of things ... But I don't know whether she was...I don't think she was a veterinary nurse who I initially spoke to. [Owner 12]

Receptionists were recalled to have performed a range of administrative roles including booking appointments over the telephone, checking owners in on arrival, weighing their pet and recording its weight, dispensing treatments, taking payment and arranging future appointments.

There's a customer scale in the waiting room. So l've just been popping him in and putting him on the scale and telling the receptionist and she's been updating his records for me so that the vet was kept informed... [Owner 11]

Some owners recalled having asked the receptionist healthcare questions that they had intended to ask the veterinary surgeon but had forgotten, or had not felt able to ask. Receptionists were therefore an important, or sole, source of information for some owners on practice policies, insurance, preventative health and topics such as feeding, weight management and behaviour.

And she gave me lots of information about how the [Pet health] scheme worked. So I discussed that with the receptionist. The vet didn't mention it. [Owner 10]

Most appeared to trust the information and advice that they had been given by the person on the reception desk. Only rarely were receptionists recalled to have provided owners with incorrect information or to have been unable to answer a question.

I did ask the receptionist for a new prescription of the wormer and the receptionist said it should only be given every six months or every three months, and I said 'Oh, that's different; I'm using it every month because I want to give him extra protection'. So she had to go and check that with the vet. [Owner 1]

The receptionist's personality was often discussed by participants. Reception staff were described as chatty, helpful, reassuring and friendly, and some owners described having long relationships with specific staff members. Receptionists telling personal stories about their own pets or recounting their own experiences of veterinary healthcare appeared to be particularly helpful in reducing anxiety for some owners. Importantly, several identified receptionists as the only practice staff member with whom they could have a conversation that was not time-pressured. 
They've got more time and it was sort of more as if you were chatting to friends valuable than some of the... you know, it wasn't all the medical aspects of things; just things to do with the experience of having a puppy, that side of it. [Owner 4]

Rapport appeared particularly good when the receptionist remembered the owner and their pet, and when the receptionist was felt to understand how important the individual pet was to that owner. A few owners identified the receptionist as key to how they and their pet felt about visiting the practice.

It's a very friendly practice. You actually feel quite welcomed and put at ease. I think that's quite nice. The dogs don't mind going in. That's the really important thing. [Owner 5]

As with the receptionists, some owners described having built relationships with veterinary nurses over a period of years. Owners appreciated advice being available from a veterinary nurse in the waiting room without the need to book an appointment; again the sense that the veterinary nurses were not rushing them was important to many.

...because the medications that she's on can make her a bit constipated so if I need any care, I can ring them up and nip, you know, to the vet nurse and they'll...they'll give me something for it so...erm...I can talk to them about pretty much anything regarding [my dog]. [Owner 6]

A few owners appeared confident that veterinary nurses could provide them with reliable pet health advice on a wide range of topics. Some had accessed veterinary nurse-led clinics following advice from a veterinary surgeon and were positive about the experience. Others were aware that clinics were available but had not used them, and a few did not think that such clinics were available in their practice.

The practice where we're at now, the vet nurses do an awful lot, and they did the blood tests and go through the worming and everything with you, which I think is really good. That frees up your time with the vet for other bits and pieces. [Owner 3]

Several owners were not sure what veterinary nurses were qualified to do, or how their role fitted with that of a veterinary surgeon. The risk that the veterinary nurse might not being able to solve a problem, necessitating a subsequent consultation with a veterinary surgeon, was the most common barrier described to attending veterinary nurse-led clinics.

I suppose there's exception that the vets are probably more there to... I don't want to... how do you put it? But obviously they're more qualified to do certain procedures so whether you would, with that exception, you'd be talking to them say, more about an operation for example whereas you might be talking...I don't know, I suppose veterinary nurses know a great deal about what they're doing... [Owner 12]

\section{Veterinary surgeons}

Veterinary surgeons described receptionists as having a wide range of roles including: provision of information about products and services; answering the telephone; triage; organising appointments; scanning microchips; weighing pets; dispensing medications; overseeing pet healthcare plans; and taking payments. Several identified their reliance on the practice receptionist to advise owners on ectoparasiticide choices, particularly in relation to pet healthcare plans. 
Definitely with puppies and kittens I discuss [parasite prevention]... Obviously if they are on the Pet Health Club, it takes care of itself. So I might ask [the owners] if they have picked the stuff up, if they are ok.... [Veterinary surgeon 6]

Others described the reception desk to be a place for owners to receive advice on topics such as diet and insurance. None voiced any doubt that their reception staff were providing correct information. Few specifically articulated a role for the receptionist in rapport building, though several activities performed by receptionists were identified as important in bonding clients to the practice.

The receptionist scans every dog that comes in for a microchip, just to make sure. And it's also, again interaction or an ice breaker with the client, just even, interact more with that client than just saying "Just sit down". And they check their details and stuff. [Veterinary surgeon 9]

All veterinary surgeons described a beneficial role for veterinary nurses in providing free receptionbased advice about pet health problems. Whilst some described specifically sending owners to talk to veterinary nurses in the waiting room, others expressed frustration that veterinary nurses were not always free. Rarely, veterinary surgeons reported that some of their practice veterinary nurses did not wish to have any form of client-facing role.

It depends very much on their personal interest because some nurses are very in to talking to clients and some aren't. [Veterinary surgeon 5]

Veterinary surgeons working in small practices or branch clinics typically felt that they did not have an adequate number of public-facing veterinary nurses to run veterinary nurse-led clinics. Sometimes, veterinary surgeons plugged the gap.

We do offer free nurse consults. [....] If we don't have enough nurses at any one time, then they will see a vet and we just won't charge them.... [Veterinary surgeon 6]

In contrast, veterinary surgeons working within larger practices described their veterinary nurses to run a wide range of preventative health clinics including 'weigh and worm' appointments for young animals, dental, mobility and weight loss clinics and in some instances conducting first or second vaccination consultations.

I often try to get, you know, if there is an animal I think has a weight issue, I try to get them straight in with the nurse immediately. Just so that they might spend half an hour in the practice rather than just 10 minutes. Or if it's an old dog, get them in with the nurse. The nurses can take bloods and such like, it is no problem for them. [Veterinary surgeon 11]

None of the veterinary surgeons discussed the need for veterinary nurses giving advice in the reception area to have received training. However, some confusion was evident about whether veterinary nurses could lead consultations.

In our practice we don't have as many qualified procedural auxiliaries so until we provide a nurse clinic or someone that's undergone training, I don't know how the Royal College would stand with advice being given by an auxiliary. I don't think they're really allowed to give advice so whoever takes the consultation is giving advice really.... [Veterinary surgeon 3]

Almost all veterinary nurse-led clinics were described to be free of charge. A few veterinary surgeons commented that uptake had been too low when there had been a fee; others said the uptake of free veterinary nurse-led clinics was still poor. Some thought owners increasingly recognised the level of training that qualified veterinary nurses had received, but others were less sure. 
I think there'd be the core of people who, probably the same people who always wanted to see the vets as well, older school people, but I think the majority of people would be quite open to nurses and I think most of them would have a lot of respect for the training they do. [Veterinary surgeon 13]

Attitudes to veterinary nurses leading, or assisting with, consultations varied between veterinary surgeons. Several expressed great confidence in their veterinary nurses' consultation skills, particularly in communicating difficult concepts. Others expressed a desire for veterinary nurses to perform certain consultation types that they didn't enjoy themselves but felt their veterinary nurses did.

I don't want to be doing weight check clinics and working out how much dog should weigh, that, you know, I know he needs to lose weight and it's just something that I don't think is a good use of my time because that is a bit of maths and then a chat gently with the client to persuade them that actually body condition score of 9 out of 9 is dreadful for a dog. It is very... it is more... I know the nurses love doing that. [Veterinary surgeon 14]

A few thought that regardless of how well qualified their veterinary nurses were, they would still prefer to perform any history taking aspects of a consultation themselves. A sense of personal responsibility for the outcome of the consultation, the likelihood that they would just repeat the same questions themselves and their personal desire to remain in charge were given as justifications.

I sometimes just rather do it myself because sometimes there's not... everyone says things differently and everyone has differing opinions as well and I kind of like my way. But I am a control freak. [Veterinary surgeon 10]

\section{Discussion}

These interviews provide an important contribution to the sparse literature on the roles of receptionists and veterinary nurses in UK small animal practice. Although the importance of reception staff in building rapport with owners has been identified, this study suggests it may not be fully recognised by all veterinary surgeons. The reliance of both owners and veterinary surgeons on the reception area as a source of pet health advice makes evident the urgent need for veterinary receptionists to be offered appropriate training. Neither owners nor veterinary surgeons were universally confident about which services veterinary nurses could, or should, provide, suggesting greater clarity is needed. Further work, involving reception and nursing staff, is needed to fully understand their roles in small animal veterinary practice.

The importance to the client experience of reception staff being friendly, un-hurried and empathic was evident in the owner interviews. Veterinary surgeons appeared less aware of this, instead placing more emphasis on their administrative roles. As a result, reception staff may face challenges in balancing provision of this client-facing role with their administrative workload. ${ }^{27}$ Parallels can be drawn with receptionists working in human healthcare settings. ${ }^{28-30}$ In these studies, receptionists identified relationships with clients to be one of their greatest sources of job satisfaction ${ }^{31}$, but reported that juggling the range of tasks expected of them with inadequate time or training was an under-valued skill. ${ }^{30}$

These interviews provide further evidence that veterinary reception staff are a key source of owner advice and information about small animal preventative medicines ${ }^{17,32}$ and broader pet health topics. ${ }^{33}$ Practices need to ensure that receptionists' training reflects their role, and practice 
managers should consider whether owners can, and should, understand the qualifications of staff providing advice. This may not be simple, as veterinary nurses and receptionists appear commonly to share reception duties in some practices. ${ }^{34}$ Furthermore, since the veterinary nursing title in the UK is unprotected ${ }^{15}$, it is not possible to determine how many of the staff described as "veterinary nurses" by our interviewees were actually registered veterinary nurses. Our work therefore supports the potential need for the statutory protection of the veterinary nursing title in the UK, and demonstrates the urgent need for further understanding and clarity of the training, roles and responsibilities of all client-facing staff within small animal veterinary practices.

Ackerman ${ }^{35}$ describes many advantages of veterinary nurses-led clinics, including bonding owners to a practice and answering questions that owners may have been too embarrassed to ask a veterinary surgeon. Those benefits were exemplified in the current study. However, challenges were evident too, some of which have been discussed in the veterinary nursing literature. ${ }^{7,36,37}$ In particular, the ability of practices to ensure suitable veterinary nurses are available when needed, given that uptake for their services can poor, may represent a considerable logistical problem. In addition, our work also builds on that by Kinnison and others ${ }^{2}$ in identifying veterinary nursing clinics as an exemplar of the difficulties associated with inter-professional working. The lack of protection of the veterinary nursing title could contribute to the lack of clarity amongst some veterinary surgeons and owners around the roles veterinary nurses can perform, and subsequently, the perceived need to give veterinary nurses' time away for free. These problems must be addressed before the full potential can be realised of veterinary nurses in a consulting role.

The wide-reaching and important role of veterinary nurses and receptionists in small animal preventative healthcare in the UK has not previously been documented. As this study did not include veterinary nurses and receptionists, it is unclear how accurately veterinary surgeon and owner perceptions of their roles match their own. Collecting those data will be an important next step and the current research provides clear justification for the inclusion of these staff in future work exploring both preventative healthcare and the client experience during interactions with veterinary practices. As with any qualitative research, extrapolation of these results beyond the study population should be performed with caution. Interviewees may have had specific motivations to be involved, and there remains the possibility that additional participants could have identified further important perspectives. ${ }^{23}$ However, we sought to avoid this by using multiple recruitment strategies, a purposive sampling frame and obtaining data saturation. All the researchers involved in this study were veterinary surgeons. Whilst this could have introduced some bias into data collection and interpretation, it was invaluable in designing the interview guides and recruiting participants.

The findings of this study should be of direct relevance to any stakeholders in small animal preventative medicine and those interested in inter-professional relationships. The role of veterinary nurses and receptionists has long been overlooked and it is hoped that their inclusion will become integral to conducting future practice-based research. Reception staff have administrative, rapport and educational roles, the complexity and importance of which should be recognised by veterinary surgeons and employers. Given their key role as an information source for owners about a wide range of pet health and preventative medicine topics, adequate training must be available. Furthermore, receptionists provide an excellent, and perhaps under-recognised route to communicate important pet health messages to owners. The lack of protection of the veterinary nursing title is clearly problematic, and both registered veterinary nurses and other staff in clientfacing roles urgently need to be included in research to better understand and define their role within veterinary practices. 


\section{Conflict of interest}

340 None.

\section{Acknowledgements}

342 The authors are grateful to all the owners and veterinary surgeons who took part in this study. The

343 Centre for Evidence-based Veterinary Medicine (CEVM) is supported by an unrestricted grant from

344 Elanco Animal Health and The University of Nottingham. The authors received additional financial

345 support from MSD Animal Health for the collection and analysis of these data. The study design,

346 analysis, interpretation of the results, decision to publish and writing of the manuscript were

347 undertaken independently of all funders of the CEVM.

\section{References}

3491 JERVING C. (1993) Do your support staff lay the golden egg? In Practice 15(4), 198-201.

3502 KINNISON T., MAY S. A., GUILE D. (2014) Inter-professional practice: From veterinarian to the 351 veterinary team. Journal of Veterinary Medical Education 41(2), 172-78.

3523 KINNISON I., GUILE D., MAY S. A. (2015) Errors in veterinary practice: Preliminary lessons for 353 building better veterinary teams. Veterinary Record 177(19), 492.

3544 KINNISON T., LUMBIS R., ORPET H., WELSH P., GREGORY S., BAILLIE S. (2011) Piloting interprofessional education interventions with veterinary and veterinary nursing students. Journal of Veterinary Medical Education 38(3), 311-18.

5 KINNISON T., MAY S. A., GUILE D. (2015) Veterinary team interactions, part one: The practice effect. Veterinary Record 177(16), 419.

6 YEATES J. W. (2014) The role of the veterinary nurse in animal welfare. Veterinary Nursing Journal 29, 250-51.

7 WILD S. (2017) Veterinary nurse clinics - opportunities and obstacles. Veterinary Nursing Journal $32(4), 118-20$.

8 JOBSON L. (2013) How to run weight clinics effectively in practice. Veterinary Nursing Journal 28(11), 372-74.

9 NELSON D., WELSH P. (2015) Using the ability model to design and implement a patient care plan. The Veterinary Nurse 6(3), 141-49.

10 LAMBERT A. (2013) Customer-centred practice - is yours? Veterinary Nursing Journal 28(1), 2425.

11 SHERIDAN L., TOTTEY H. (2017) A compassionate journey part 3: The client experience. The Veterinary Nurse 8(2), 66-73.

12 HAMLIN J. (2013) Are VNs professionals or paraprofessionals? The Veterinary Nurse 4(6), 315. 13 ANON. (2018) Code of professional conduct for veterinary nurses. https://www.rcvs.org.uk/setting-standards/advice-and-guidance/code-of-professional-conduct-forveterinary-nurses/ Accessed February 27, 2018

14 ANON. (2018) About the VN Register. https://www.rcvs.org.uk/registration/check-theregister/about-the-vn-register/ Accessed February 27, 2017

15 ANON. (2016) RCVS responds to DEFRA statement on its 'Protect the VN title' petition. https://www.rcvs.org.uk/news-and-views/news/rcvs-responds-to-defra-statement-on-its-protectthe-vn-title/ Accessed August 17, 2017

16 LAMBERT A. (2015) The equine reception experience. In Practice 37(2), 96-98.

17 RAVETZ G. (2017) Prevention is better than cure: Promoting pet health plans. Veterinary Business Journal 170, 16-19.

18 TONG A., SAINSBURY P., CRAIG J. (2007) Consolidated criteria for reporting qualitative research (COREQ): A 32-item checklist for interviews and focus groups. International Journal for Quality in Healthcare 19(6), 349-57. 
19 BRYMAN A. (2012) Sampling in qualitative research. In Social Research Methods. $4^{\text {th }}$ ed. Oxford University Press. p. 415-29.

20 ROBINSON N.J., BRENNAN M.L., COBB M., DEAN R.S. (2016) Investigating preventive-medicine consultations in first-opinion small-animal practice in the united kingdom using direct observation. Preventive Veterinary Medicine 124, 69-77.

21 BRAUN V., CLARKE V. (2006) Using thematic analysis in psychology. Qualitative Research in Psychology 3(2), 77-101.

22 BELSHAW Z., ROBINSON N.J., DEAN R.S., BRENNAN M.L. (2018a) "I always feel like I have to rush..."Pet owner and small animal veterinary surgeons' reflections on time during preventative healthcare consultations in the United Kingdom Veterinary Sciences 5, 20.

23 ZIEBLAND S., MCPHERSON A. (2006) Making sense of qualitative data analysis: An introduction with illustrations from dipex (personal experiences of health and illness). Medical Educcation 40(5), 405-14.

24 CLARKE V., BRAUN V. (2013) Successful Qualitative Research: A Practical Guide for Beginners. 1st ed. Sage Publications Limited.

25 BELSHAW Z., ROBINSON N.J., DEAN R.S., BRENNAN M.L. (2018b) Owners and veterinary surgeons in the United Kingdom disagree about what should happen during a small animal vaccination consultation. Veterinary Sciences 2018, 5, 7.

26 BELSHAW Z., ROBINSON N.J., DEAN R.S, BRENNAN M.L (2018c) Motivators and barriers for dog and cat owners and veterinary surgeons in the United Kingdom to use preventative medicines. Preventive Veterinary Medicine In press.

27 FAULKNER B. (2014) First impressions count: Training the receptionist. Veterinary Business Journal 137, 15.

28 NEUWELT P. M., KEARNS R. A., BROWNE A. J. (2015) The place of receptionists in access to primary care: Challenges in the space between community and consultation. Social Science and Medicine 133, 287-95.

29 NEUWELT P. M., KEARNS R. A., CAIRNS I. R. (2016) The care work of general practice receptionists. Journal of Primary Health Care 8(2), 122-9.

30 HAMMOND J., GRAVENHORST K., FUNNELL E., BEATTY S., HIBBERT D., LAMB J., and others. (2013) Slaying the dragon myth: An ethnographic study of receptionists in UK general practice. British Journal of General Practice 63(608), e177-84.

31 EISNER M., BRITTEN N. (1999) What do general practice receptionists think and feel about their work? British Journal of General Practice 49(439), 103-6.

32 WRIGHT I. (2017) Nurse-led parasite control. The Veterinary Nurse 8(2), 60-4.

33 ROSHIER A. L., MCBRIDE E. A. (2013) Veterinarians' perceptions of behaviour support in smallanimal practice. Veterinary Record. 172(10), 267

34 SHILCOCK M. (2006) Are you getting your message across? Veterinary Nursing Journal 21(10), 12. 35 ACKERMAN N. (2012) The consulting veterinary nurse. Veterinary Nursing Journal 27(7), 269-70. 36 SPENCER C. (2010) Easy steps to conducting a nurse consultation. Veterinary Nursing Journal 25(4), 21-52.

37 MACDONALD H. (2011) Maximising your team potential. Veterinary Nursing Journal 26(1), 28-9. 